\title{
Raman spectroscopy revealing noble gas adsorption on single-walled carbon nanotube bundles
}

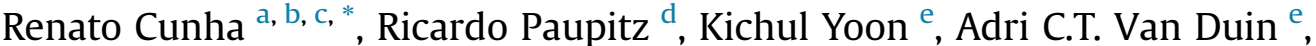 \\ Ana Laura Elías ${ }^{\text {a, b }}$, Victor Carozo ${ }^{\text {a, b, k, Archi Dasgupta }}{ }^{\text {, }}$ Kazunori Fujisawa ${ }^{\text {a, b }}$, \\ Néstor Perea Lopez a, b. Paulo T. Araujo g, h, **, Mauricio Terrones a, i, b, j, f, ,*** \\ a Department of Physics, The Pennsylvania State University, University Park, PA, 16802, USA \\ ${ }^{\mathrm{b}}$ Center for 2-Dimensional and Layered Materials, The Pennsylvania State University, University Park, PA, 16802, USA \\ ${ }^{\mathrm{c}}$ Faculdade de Ciências Exatas e Naturais, Universidade Federal do Pará, Campus Universitário de Tocantins, PA, 68.400-000, Brazil \\ ${ }^{\mathrm{d}}$ Departamento de Física, IGCE, Universidade Estadual Paulista, UNESP, 13506-900, Rio Claro, SP, Brazil \\ e Department of Mechanical and Nuclear Engineering, The Pennsylvania State University, University Park, PA, 16802, USA \\ ${ }^{\mathrm{f}}$ Department of Chemistry, The Pennsylvania State University, University Park, PA, 16802, USA \\ ${ }^{g}$ Department of Physics and Astronomy, The University of Alabama, Tuscaloosa, AL, 35487, USA \\ h Center for Materials for Information Technology (MINT Center), The University of Alabama, Tuscaloosa, AL, 35487, USA \\ i Department of Materials Sciences and Engineering, The Pennsylvania State University, University Park, PA, 16802, USA \\ j Institute of Carbon Science and Technology, Shinshu University, 4-17-1 Wakasato, Nagano-city, 380-8553, Japan \\ ${ }^{\mathrm{k}}$ Department of Physics, Pontifical Catholic University of Rio de Janeiro, Rio de Janeiro, RJ 22451900, Brazil
}

\section{A R T I C L E I N F O}

\section{Article history:}

Received 19 May 2017

Received in revised form

3 October 2017

Accepted 5 November 2017

Available online 7 November 2017

\section{Keywords:}

Adsorption

Raman spectroscopy

Simulation

\begin{abstract}
A B S T R A C T
The interaction of the noble atoms ( $\mathrm{Ar}$ and $\mathrm{Xe}$ ) with single-walled carbon nanotube (SWCNT) bundles are investigated using Raman spectroscopy in conjunction with computational modeling known as ReaxFF force field. SWCNT bundles were deposited on transmission electron microscopy (TEM) grids, and different noble gases were adsorbed onto the nanotubes at $20 \mathrm{~K}$. Raman spectra acquired show significant frequency blueshifts of the radial breathing mode (RBM), G- and $\mathrm{G}^{\prime}$ (or 2D)-bands due to gas solidification within the external groove sites (free spaces between the tubes in the bundle) and external surfaces of the bundles. This solid shell formed by the adsorbed gases contributes with a hydrostatic pressure to the system. We show from Raman measurements that the frequencies found after gas adsorption exhibit almost the same shifts indicating that the interactions between SWCNTs bundles and the gases ( $\mathrm{Ar}$ or $\mathrm{Xe})$ are nearly identical.
\end{abstract}

๑) 2017 Elsevier Ltd. All rights reserved.

\section{Introduction}

Single-walled carbon nanotubes (SWCNTs) have attracted intense research interest in the past 20 years [1,2]. These materials due to their unique structure and remarkable properties have been used in a wide variety of applications [3,4]. Particularly, SWCNTs

\footnotetext{
* Corresponding author. Department of Physics, The Pennsylvania State University, University Park, PA, 16802, USA.

** Corresponding author. Department of Physics and Astronomy, The University of Alabama, Tuscaloosa, AL, 35487, USA.

*** Corresponding author. Department of Physics, The Pennsylvania State University, University Park, PA, 16802, USA.

E-mail addresses: jrecunha@gmail.com (R. Cunha), paulo.taraujo@ua.edu (P.T. Araujo), mut11@psu.edu (M. Terrones).
}

could have applications in nanoelectronic devices [5], gas storage [6], sensor [7], medical technologies [8], and also as novel porous materials for the adsorption of gases [9]. SWCNTs grow in bundles which are held together due to strong van der Waals interactions [10], and this process creates new types of adsorption sites which are absent in an individual SWCNT, which counts solely with the tube's surface. These new adsorption sites make bundles considerably more reactive to external agents, which could be easily adsorbed to the bundles' effective surface when compared to isolated SWCNTs [11,12].

The adsorption of noble gases on SWCNTs is highly important, as these noble species constitute model systems of physisorption, in which atoms are weakly bound to the surface [13,14]. SWNTs exhibit high surface areas and precisely defined pores, making them very useful materials for gas adsorption and purification [15]. 
The inertness of the noble gases typically excludes the possibility of chemical and polar interactions with the surface, and exclusively results in physical absorption mediated by weak surface interactions. A large number of theoretical and experimental studies have been carried out on adsorption of noble gases on various CNTs, single- or multi-walled (closed- or open-ended) [15-19]. Most of the studies on modeling the interactions between atoms and molecules with CNTs are based on density functional theory (DFT) calculations [20], molecular dynamics (MD) [21], and Monte Carlo simulations [22]. However, detailed experimental studies using Raman spectroscopy at low temperature have not been reported yet. It is well known that bundles of carbon nanotube exhibit a distribution of tube diameters [11]. Four different adsorptions sites have been identified on heterogeneous bundles of SWNTs [21]: (1) interstitial channels between three adjacent tubes (IC sites); (2) groove sites ( $G$ sites); (3) surface sites ( $\mathrm{S}$ sites) on the curved outer surface of the bundles; and (4) open-ended SWNT bundles within interior adsorption sites (INT). However, the determination of the number of available INT, IC, G, and S sites is a challenging task [23]. Due to their stable thermodynamic and mechanical properties, and also their uniform porosity distribution, SWCNTs have been considered as excellent candidates for gas separation applications [15]. SWCNTs have also been utilized as gas sensors since their electronic properties change dramatically and selectively upon gas adsorption [24,25]. SWCNTs, however, present a drawback: different types of tubes present different behaviors regarding their electronic and vibrational structures and, to the best of the author's knowledge, growth of unique CNT species with a particular set of properties is still under development. This fact jeopardizes the application of carbon nanotubes in a large scale.

In the present work, Raman spectroscopy was used to investigate the adsorption of noble gases on SWCNTs bundles at low temperature $(20 \mathrm{~K})$. Here, we interpret the shifts in the SWCNT phonon frequencies observed in the Raman spectra due uniform compressions related to Argon ( $\mathrm{Ar}$ ) and xenon (Xe) atoms adsorbed at the surface of the SWCNT bundles. Raman spectroscopy is one of the most sensitive, informative and also nondestructive spectroscopic methods to analyze nanostructures, particularly carbon nanostructures $[26,27]$. This technique has proven itself very successful when monitoring phonons in a variety of graphitic materials including graphite, graphene, fullerenes and SWCNTs [28,29]. Here, significant frequency blueshifts of $\sim 4 \mathrm{~cm}^{-1}$ for the Raman RBMbands were observed for both the Ar/SWCNTs and Xe/SWCNTs at $20 \mathrm{~K}$, which reveal strong evidence of gas adsorption on the bundled carbon nanostructure. It is worth mentioning that, on the other hand, no significant changes were observed in the mode's linewidth and intensity. The observation of unchanged linewidths and intensities along with the fact that noble atoms are hard to be ionized to receive (donate) electrons from (to) carbon nanotubes, give us a clue that doping is not the reason why the frequency shifts are taking place [30-36]. Moreover, these gas molecules are most often weakly bound to the nanotube structures. Thus, we hypothesize that such weak tube-adsorbate interaction can be well described in terms of surface physisorption, which would uniformly compress the nanotubes. Molecular dynamics simulations seem to agree well with our Raman measurements and, therefore, our explanation hypothesis.

\section{Methods}

\subsection{Experimental section}

SWCNTs with an average diameter between 1.3 and $1.5 \mathrm{~nm}$ were synthesized using the arc discharge method and used as received (CARBOLEX Inc.). These SWCNTs were sonicated in an aqueous solution for 100 min in five cycles of 20 min each, so that overheating could be avoided. The sonication was performed using an ultrasonic tip processor (at $18 \mathrm{~W}$ of power) so that the size of the bundles is reduced. A few drops of the solution were deposited on a transmission electron microscopy (TEM) grid. Each grid was left at ambient conditions until all the water was fully evaporated. Fig. 1 presents high-resolution transmission electron microscopy (HRTEM) images of the SWCNTs bundles acquired with a Joel 2010F electron microscope operating with an accelerating voltage of $200 \mathrm{kV}$ (field-emission source, ultra-high resolution pole piece ( $\mathrm{Cs}=0.5 \mathrm{~mm}$ ), $1.9 \AA$ Scherzer limit). By utilizing the TEM images from our samples, we can estimate the bundle size to be on average $14.6 \mathrm{~nm}$ with a standard deviation of $6.2 \mathrm{~nm}$. Given that the diameter of these bundles is about 10 times larger than an individual SWCNT, the estimated surface area should be around $300 \mathrm{~m}^{2} / \mathrm{g}$, which is in reasonable agreement with the measured BET surface area (see Fig. S1 in the supplementary information). Note that it is difficult to determine accurately the number of tubes within a bundle, but the tube configuration can be studied in detail using Raman spectroscopy and Molecular dynamics simulations.

The experimental set-up consists of a liquid helium cryostat in which the TEM grid containing the SWCNT bundles, is placed. This plate is pumped with a mechanical-turbo pump. The gas adsorption was performed at $20 \mathrm{~K}$ and the gas flow was accurately controlled by a mass flow controller (MFC). At this point, Raman spectroscopy on both SWCNTs bundles was performed using a Renishaw inVia microscope-based Raman spectrometer. The spectra were collected in a backscattering geometry using three different excitation wavelengths: $514.5 \mathrm{~nm}(2.41 \mathrm{eV}), 488 \mathrm{~nm}$ $(2.54 \mathrm{eV})$, and $785 \mathrm{~nm}(1.58 \mathrm{eV})$. The incident beam was focused through the windows of the chamber onto the sample surface with a microscope objective (Olympus $50 \times$ ) and the back-scattered light was collected with the same objective. The measurements were performed using very low laser power densities. After several tests, the excitation laser power density was found adequate for magnitudes $<0.3 \mathrm{~mW} / \mu \mathrm{m}^{2}$. Such low power densities were enough to avoid the effect of laser-heating. In addition, no laser power dependence of the Raman spectra of SWCNTs was observed for power densities $<0.3 \mathrm{~mW} / \mu \mathrm{m}^{2}$. The schematics of the experimental set-up are shown in the Fig. 2. For the measurements at $20 \mathrm{~K}$ under vacuum, the MFC is closed and the pressure inside the chamber is kept around $10^{-6}$ Torr, whereas for the measurements with gas adsorption, the valve of the vacuum pump is closed and the MFC is opened for a few seconds so that the gas is slowly admitted into the chamber increasing the pressure to $\sim 10^{-3}$ Torr.

Finally, all of our spectra were carefully fitted with Lorentzian curves after appropriate baselines were taken. No treatments of the raw data, such as smoothening or interpolation/extrapolation were performed. All the frequency values reported here were extracted from the fitting results. Moreover, the fitting procedure was repeated several times in order to increase accuracy. On top of that, the spectrometer we used can measure frequencies with an accuracy of $\pm 0.3 \mathrm{~cm}^{-1}$. Therefore, even though the shifts we observe are small, they are real and are consistent.

\subsection{Computational details}

In order to improve our understanding on the influence of a noble gases on the CNT vibrational modes, we simulated the essential features of our experiment. The interaction between all atoms in the system was described using a reactive methodology, the so called ReaxFF force field [37,38].

ReaxFF is a reactive force field method developed by van Duin, Goddard III and co-workers, which allows the realization of molecular dynamics simulations in which chemical reactions can 

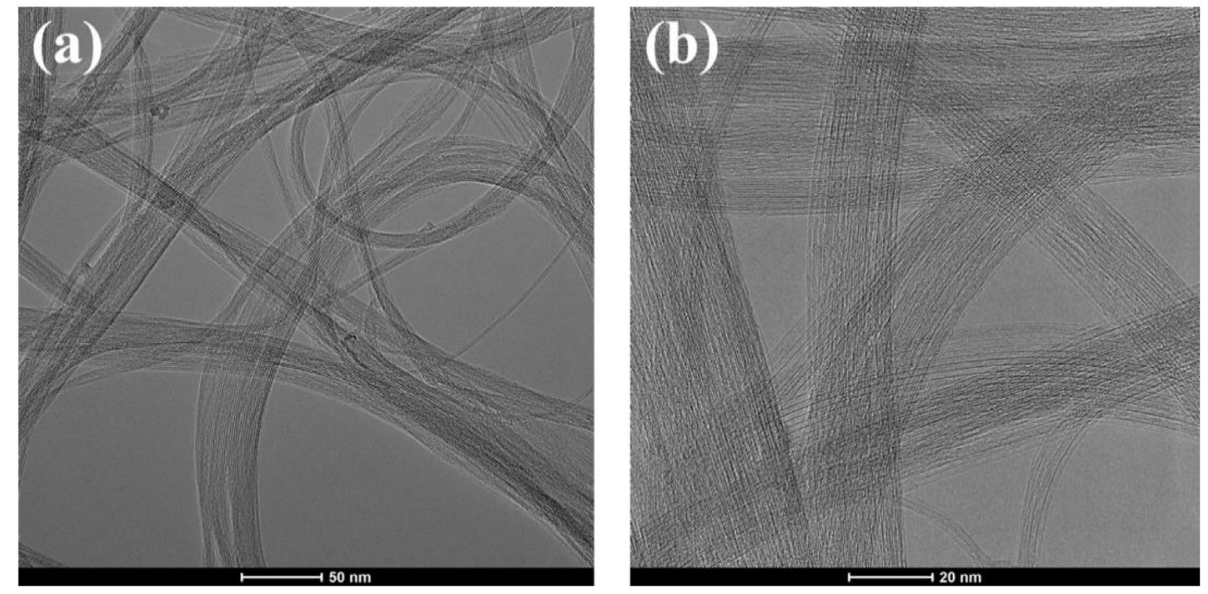

Fig. 1. (a and b) HRTEM images of the SWCNTs bundles on TEM grids.

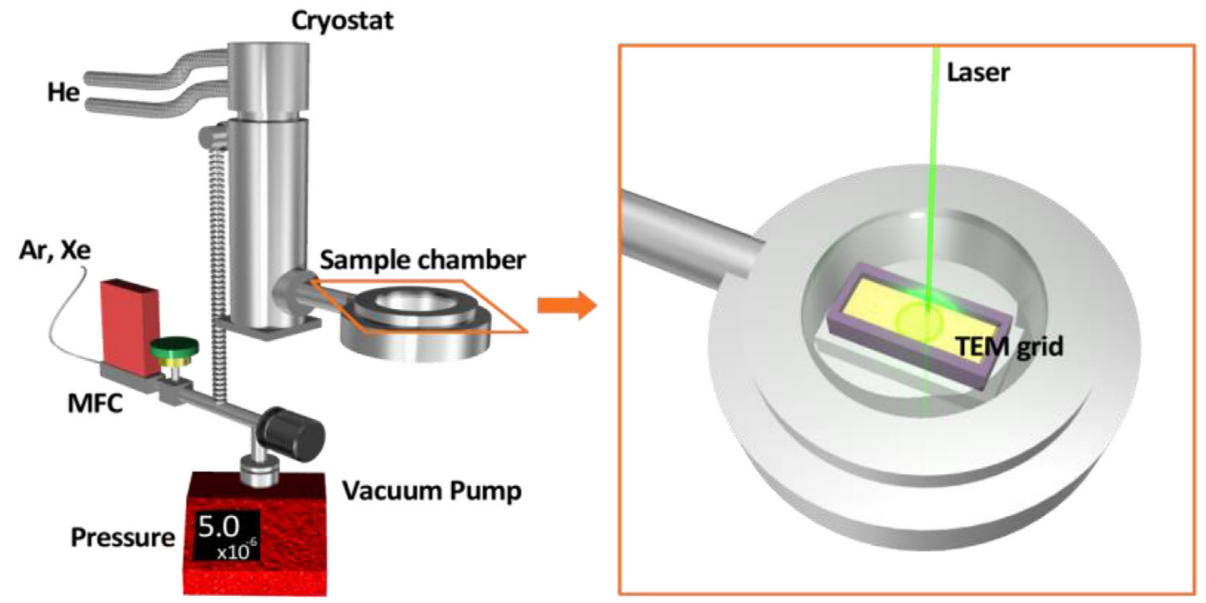

Fig. 2. Schematic set-up of the experiment showing gas admission into the chamber at law temperature. (A colour version of this figure can be viewed online.)

occur [37]. ReaxFF has some features that are similar to those found in standard non-reactive force fields. For instance, the energy of the system is divided into several contributions including valence angles, bond stretching, bending as well as non-bonded van der Waals and Coulomb interactions. The difference in comparison to nonreactive force fields resides in the fact that with this force-field, it is possible to describe the bond formation and dissociation (making/breaking bonds) as a function of bond order values. The parameters in the ReaxFF are optimized against DFT calculations and have been widely used in the description of large systems which include carbon atoms as well as other chemical elements [38]. The parameters used in the present work were specially tailored in order to describe the interaction of carbon atoms with noble gases. Molecular Dynamics simulations for low temperatures were carried out using the well-known Verlet algorithm, while the temperatures were controlled with the aid of a Nose-Hoover Thermostat, as implemented in LAMMPS software [39]. Visualizations and images used to assemble the movies (see the Supplementary Materials) were constructed using VMD software [40].

In the software we are using for the calculations, the Grand Canonical Monte Carlo simulation (properly coded) is not a feature to use when considering the specific reactive potential (ReaxFF). In order to circumvent this problem, we carried many simulations starting with different positions and random number of generator seeds, but with the same particle density and temperature. With these modifications, our results indicate that the final configurations are similar and the effect on vibrational frequencies remains the same.

\section{Results and discussion}

Fig. 3(a) shows the Raman spectra from SWCNTs bundles suspended on a TEM grid under vacuum $\left(10^{-6}\right.$ Torr $)$ at $20 \mathrm{~K}$ before and after the noble gases adsorption using a $514.5 \mathrm{~nm}(2.41 \mathrm{eV})$ excitation wavelength. As labelled in Fig. 3(a), the most notable features of the spectra are the hydrostatic breathing mode (RBM), the defect-related D-band, the G-band and the $\mathrm{G}^{\prime}$ (or 2D)-band. The RBM at 156.1 and $178.2 \mathrm{~cm}^{-1}$, highlighted in Fig. 3(b) are upshifted by $\sim 2 \mathrm{~cm}^{-1}$ after Ar adsorption. In the G-band region, the higherfrequency $\mathrm{G}^{(+)}$Raman peak, shows a smaller upshift of $\sim 1 \mathrm{~cm}^{-1}$ while the $G^{(-)}$presents a upshift of $\sim 2 \mathrm{~cm}^{-1}$ [highlighted in Fig. 3(c)]. It is interesting to note that both the RBM-band and the $\mathrm{G}^{(-)}$features are diameter dependent and since they are most affected by the adsorption, uniform compression hypothesis gain some force here. The $\mathrm{G}^{\prime}$ (or 2D)-band at $2684 \mathrm{~cm}^{-1}$, as highlighted in Fig. 3(d), is found to upshift by $\sim 2 \mathrm{~cm}^{-1}$. The D-band at $1339.7 \mathrm{~cm}^{-1}$ is weak indicating a relatively defect-free tube and this D-band undergoes negligible upshift after gas adsorption. Note that, both the D- and the $\mathrm{G}^{\prime}$ (or 2D)-band are inter-valley second order Raman scattering process which involves, respectively, one defect and one 
(a)

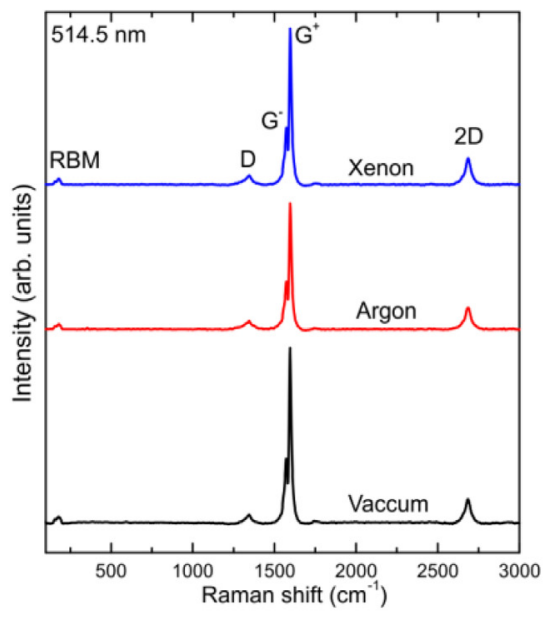

(c)

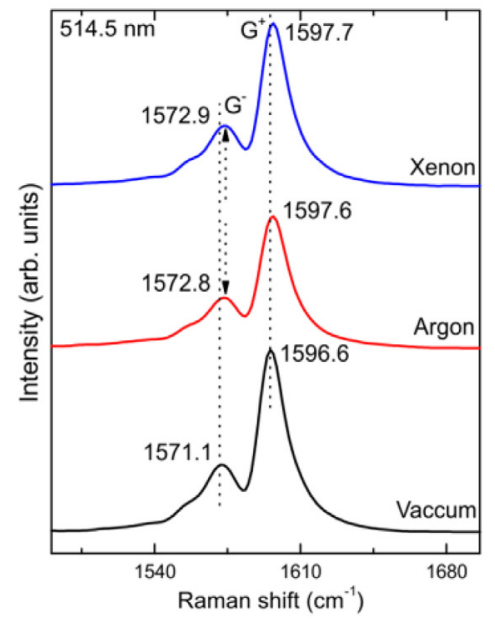

(b)

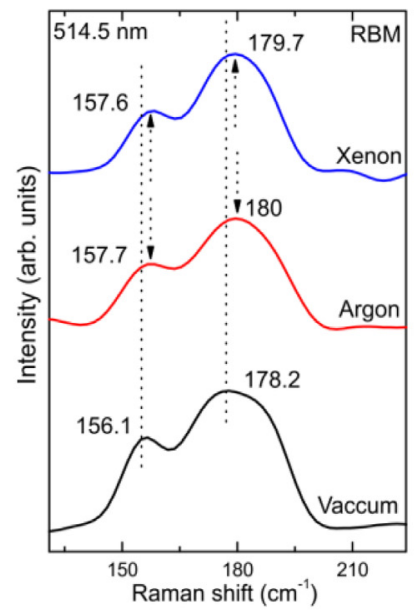

(d)

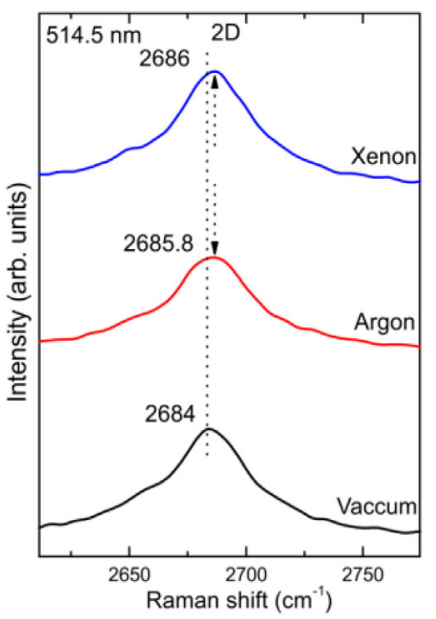

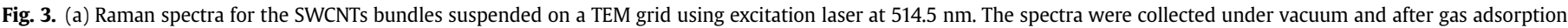

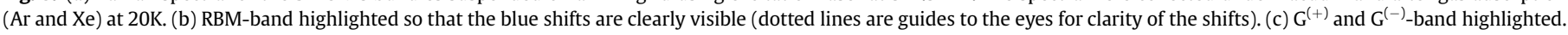
(d) $\mathrm{G}^{\prime}$ (or 2D)-band highlighted. (A colour version of this figure can be viewed online.)

phonon and two identical phonon that scatter resonantly to connect inequivalent $\mathrm{K}$ and $\mathrm{K}^{\prime}$ points back and forth [41]. These phonons are zone-edge phonons, which occur around the $\mathrm{K}$ or $\mathrm{K}^{\prime}$ points in the Brillouin zone and they are, therefore, more sensitive to perturbations than zone center phonons, which occur around the $\Gamma$ point in the Brillouin zone, as it is the case for the RBM- and Gbands.

Indeed, second order processes may be more sensitive to perturbations because: (1) elongation (shortening) of the carboncarbon bonds, which weakens (strengthens) the bonds, will downshift (upshift) the phonon's vibrational frequency. Second order processes involve twice the same phonon with opposite momentum in order to conserve momentum. In other words, if a phonon has frequency of $1000 \mathrm{~cm}^{-1}$ and the frequency changes to $1010 \mathrm{~cm}^{-1}\left(\Delta \omega=10 \mathrm{~cm}^{-1}\right)$, which means it is increased by $0.01 \%$, such increase of $0.01 \%$ would signify $\Delta \omega=20 \mathrm{~cm}^{-1}$ for a process involving twice such a phonon; (2) for intervalley scattering processes, additional shifts in the frequency can also occur due to relative changes in the slopes of the electronic dispersions around the $\mathrm{K}$ and $\mathrm{K}^{\prime}$ points, which also change the phonon momentum (changing, therefore, the phonon energy) so conservation can be attained. Such relative changes are not important for first order or intravalley processes in which either $\mathrm{K}$ or $\mathrm{K}^{\prime}$ is involved; (3) any relative motion of the $K$ and $\mathrm{K}^{\prime}$ points themselves can also change the phonon momentum, which would also add extra shifts to the frequency, and (4) the Kohn anomaly observed in the iTO phonon at the $\mathrm{K}$ point, associated to the $\mathrm{D}$ - and $\mathrm{G}^{\prime}$ (or 2D)-bands, magnifies the variations in the phonon frequency with changing the phonon momentum.

However, if our hypothesis is valid, and a uniform pressure (a hydrostatic pressure) is taking place, the RBM- and $\mathrm{G}^{(-)}$-bands should indeed be the ones most affected by the adsorption since these two modes are directly related to the tube diameters. In fact, by monitoring the Raman frequencies we see that our experiment corroborates the explanation above and lead us to deduce that the adsorbents will form an external shell surrounding the suspended bundles and, as already discussed in the literature [42-51], this shell contributes with a hydrostatic pressure to the system. Since the hydrostatic pressures may both, harden the carbon bonds and also decrease diameters, the frequencies of the RBM and $\mathrm{G}^{(-)}$-bands 
must increase as demonstrated by the experiment and supported by our calculations [42-51]. The $\mathrm{G}^{\prime}$ (or 2D)-band is also very sensitive to any changes to the vibrational and electronic structures and it also have a significant dependence with the inverse of tube diameter as well (not as strong as the dependences for the RBMand $\mathrm{G}^{(-)}$-band though) [52,53]. Again, hydrostatic pressures must, therefore, upshift the mode frequency, which agrees well with our observations [see Fig. 3(d)]. The $\mathrm{G}^{(+)}$mode is confined to the direction of the tube axis which makes it more insensitive to tube diameters' changes and less susceptible to bond hardenings due to hydrostatic pressures (provided that such pressures are much smaller than $1 \mathrm{GPa}$, which are the cases of small loads applied by AFM tips [54,55] and pressure naturally applied by the nanotubes environment [42-44], which explains the low frequency upshifts observed to this mode (see Fig. 3(c)). In fact, the environment (that is, the adsorbed atoms) likely makes a rigid cylinder around the tubes and the cylinder walls are responsible for applying the pressures. Such mechanism was discussed by Araujo et al. [42-44].

The frequencies found after Xe adsorption at the same conditions [see Fig. 3(b)-(d)] exhibit almost the same shifts indicating that the interactions between SWCNTs bundles and either Ar or Xe gases are very close at low temperatures and the difference of masses between the two atoms seems not to be very important to describe the behavior of the frequencies upshifts. The masses differences will likely manifest in temperature-dependent experiments, in which the temperatures are increased from $20 \mathrm{~K}$ to higher values. Indeed, the bigger the mass the harder the atoms start to acquire enough kinetic energy to overcome potential energy keeping them at the adsorption sites [56-58].

In order to accurately interpret our experiments, we conducted
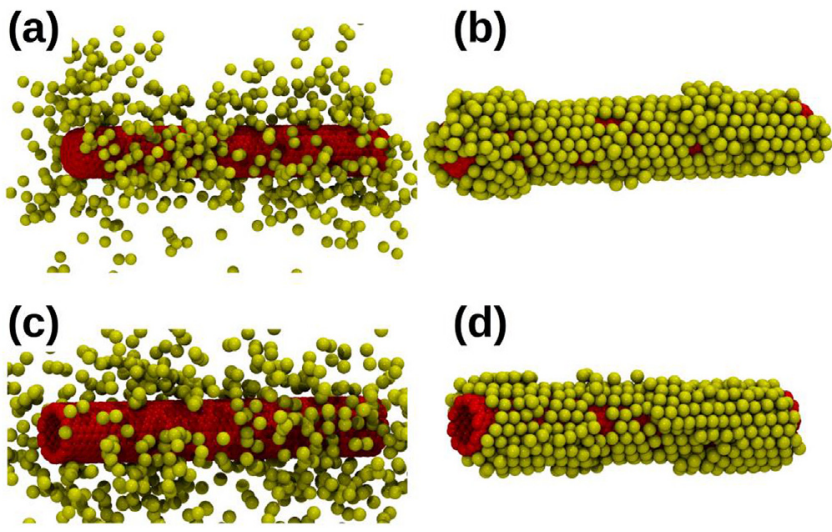

(d)

(e)

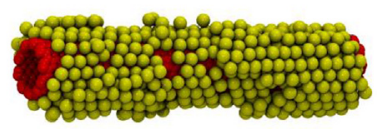

(f)
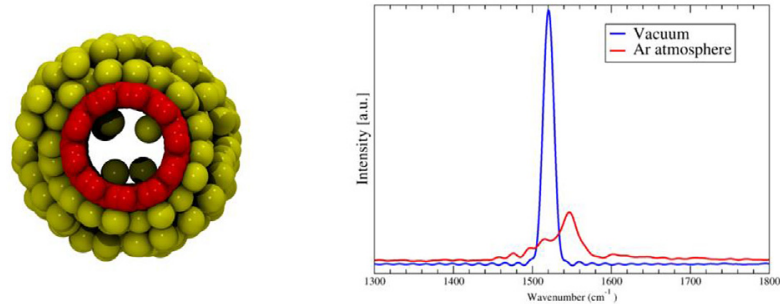

Fig. 4. Atomistic computational model used in the study of CNTs under the influence of a Noble gas atmosphere. Red balls represent carbon atoms while yellow balls represent the noble gas atoms. Representative snapshots of the initial stages of simulations carried out at $20 \mathrm{~K}$ are shown in (a) and (c) for a closed and an open CNT respectively. (b) and (d) show the final configuration of simulations initiated as shown in (a) and (b). In (e), Ar atoms present in the internal region of the opened CNT and Ar layers on the external surface. (f) Srepresentative shifting of frequency peaks caused by $\mathrm{Ar}$ adsorption on the CNT exterior surface. (A colour version of this figure can be viewed online.) simulations based on ReaxFF force field atomistic computational models as illustrated in Figs. 4 and 5. Using the adsorption model as represented in Fig. 4(a)-(e), we were able to simulate and study the behavior of an isolated CNT at $20 \mathrm{~K}$ in vacuum as well as immersed in a noble gas atmosphere. In Fig. 4, representative snapshots of the simulation conducted are shown. Namely, Fig. 4(a)-(c) show the initial snapshots of closed and open-ended CNTs, respectively, while $4(\mathrm{~b})$ and $4(\mathrm{~d})$ show the final snapshots of the same simulations, that considered $(10,10)$ CNTs immerse in $\mathrm{Ar}$ atmosphere. Fig. 4(e) illustrates the cross-section of the open-ended CNT studied, evidencing the presence of some atoms inside the tube and also demonstrating the adlayer formation of frozen Ar on the external surface of the nanotube. Fig. 4(f), on the other hand, shows a representative shifting in the frequency peaks due to Ar adsorption at low temperatures, when the system was cooled down to $20 \mathrm{~K}$.

Our calculations included also the dynamics of CNT bundles, as illustrated in Fig. 5, in vacuum and in noble gas atmospheres. In both, single CNT or CNT bundle systems we observed an upshift in the most relevant frequency peaks after the adsorption of a noble gas on the nanotube walls and interstitial sites. Naturally, these frequencies upshift will be larger for higher pressures applied by the gas adlayer. In the case of a bundle, our simulations show that there is a higher coverage of the noble gas atoms on the external grooves and on external CNTs, in complete accordance with the hypotheses considered earlier in the text. The detailed formation of this gas coverage on a CNT and on a bundle can be seen in two videos included in the supplementary materials (Figs. S3 and S4).

It is worth mentioning that both the experimental and theoretical studies suggest that at $20 \mathrm{~K}$, the Raman frequencies are upshifted due the gas solidification in the external grooves sites and external surfaces of the carbon nanotube species in the bundle. The shifts obtained are consistent with physical adsorption in which the interactions adsorbate/SWCNTs arise from van der Waals forces.

Fig. 6 shows Raman spectra of the same SWCNT bundles suspended on a TEM grid were collected in a backscattering geometry

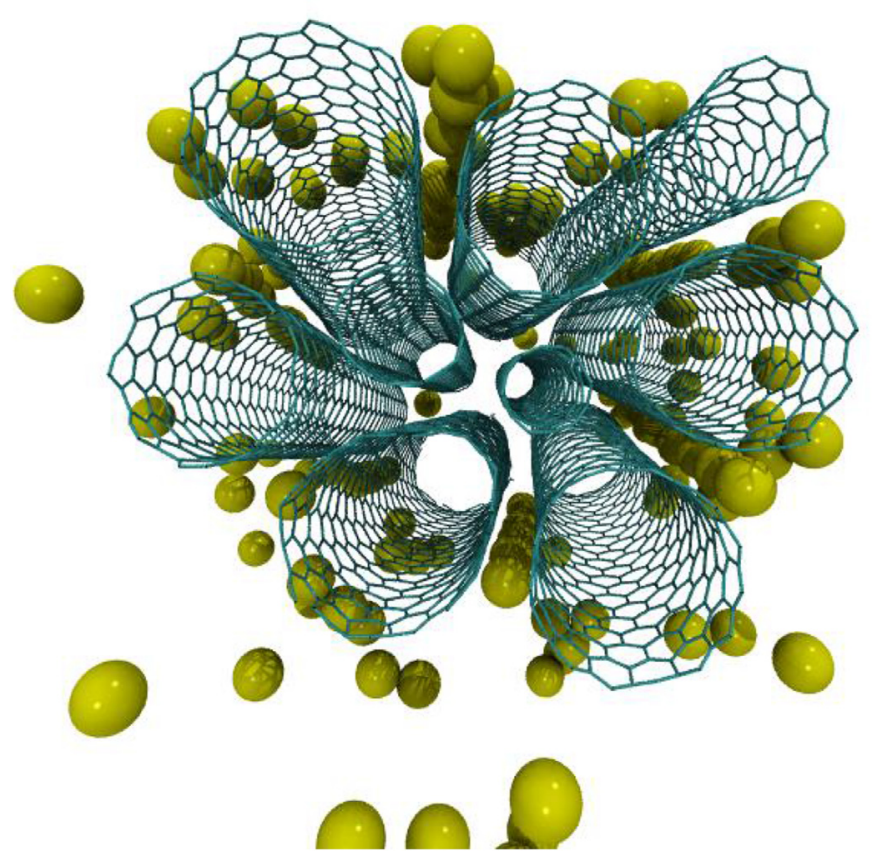

Fig. 5. Representation of SWCNTs bundles containing a distribution of different nanotube diameters. The yellow spheres represent the noble gases adsorbed on both external groove sites and external surfaces of the bundles. (A colour version of this figure can be viewed online.) 
(a)

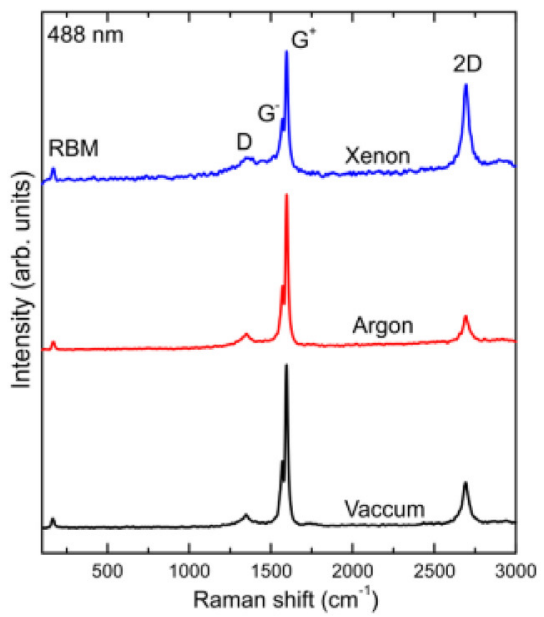

(c)

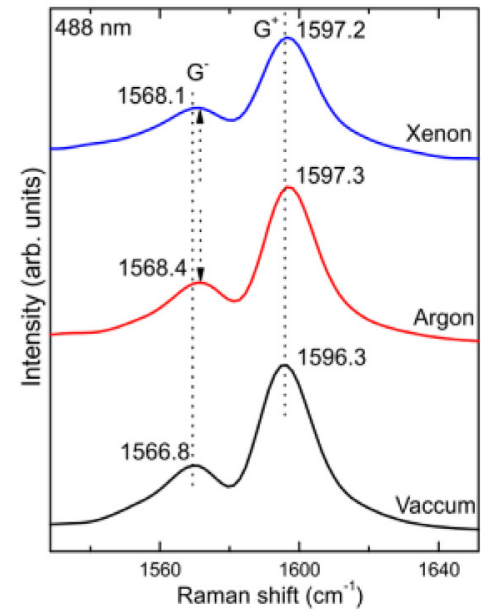

(b)

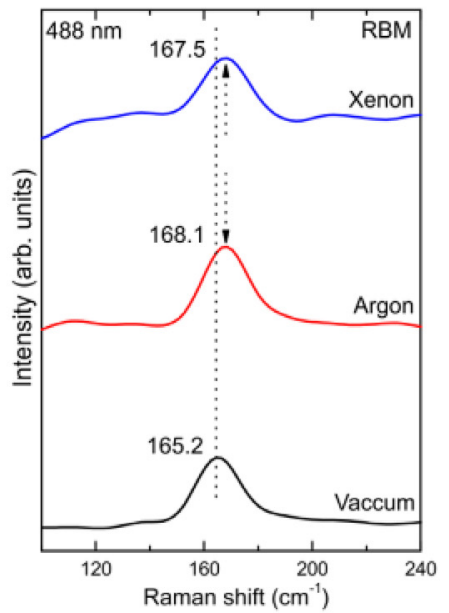

(d)

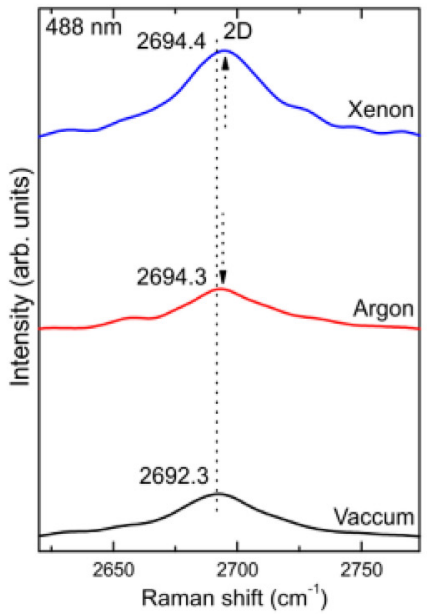

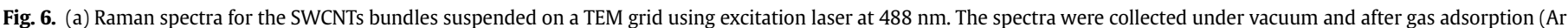

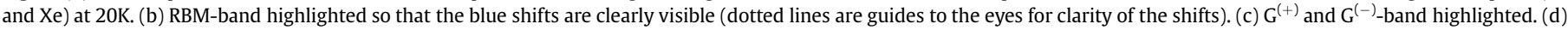
$\mathrm{G}^{\prime}$ (or 2D)-band highlighted. (A colour version of this figure can be viewed online.)

using a different excitation wavelength, namely, $488 \mathrm{~nm}(2.54 \mathrm{eV})$. Fig. 6(a) represents the RBM-, D-, G- and G'(or 2D)-bands under vacuum and after the noble gas adsorption ( $\mathrm{Ar}$ and $\mathrm{Xe}$ ) at $20 \mathrm{~K}$. Fig. 6(b)-(c) and 6(d) zoom into the RBM-, G- and $\mathrm{G}^{\prime}$ (or 2D)-bands, respectively. The RBM Raman peak at $165.2 \mathrm{~cm}^{-1}$ is upshifted equally (by $\sim 3 \mathrm{~cm}^{-1}$ ) for both $\mathrm{Ar}$ and Xe adsorption and this upshift is bigger than that observed in Fig. 3. It is well known that nanotube bundles contain a distribution of different nanotube diameters and in this case the excitation laser energy is in resonance with a tube probably located on the external surface of the bundle. These tubes exhibit higher gas coverage and therefore greater upshift in the RBM frequency. In general, we observe that regardless of the laser excitation, the behavior observed for the Raman features, the RBMband, the $G^{(-)}$- and $G^{(+)}$-bands and the $G^{\prime}$ (or 2D)-band, are consistent with the theoretical discussion presented above for carbon nanotube systems under hydrostatic pressures. This observation is important because we gain the capability of selectively probing different regions of the bundles and, therefore, probing different sites of adsorption.

Note that an interesting resonant effect is observed after Xe is adsorbed at the SWCNTs at $20 \mathrm{~K}$ (see Fig. 6(a)). Compared to the vacuum and $\mathrm{Ar}$ adsorption at $20 \mathrm{~K}$ cases, we see that the $\mathrm{G}^{\prime}$ (or 2D)band is more intense for the Xe adsorption case. In fact, Xe in the solid phase has the highest dielectric constant when compared to $\mathrm{Ar}$ and $\mathrm{Kr}$ in the solid phases, which have similar dielectric constants [59]. It is know that the energy transitions of SWCNTs, which are excitonic in nature, decrease (increase) as the dielectric constant of its environment increases (decreases). This effect is well discussed by Araujo et al. [43-45]. Therefore, the authors believe that this dielectric constant difference between Xe and the other two systems ( $\mathrm{Ar}$ and $\mathrm{Kr}$ ) is what triggers this resonant enhancement. Xe happens to offer the environment that lowers the transition energies enough to bring the SWCNT observed into a strong resonant regime with the $488 \mathrm{~nm}$ laser line. On the other hand, $\mathrm{Ar}$ and $\mathrm{Kr}$ do not lower the transition energies enough to bring the SWCNT observed to a strong resonant regime with the same laser line.

As a proof of concept we excite the bundles with an excitation wavelength of $785 \mathrm{~nm}(1.58 \mathrm{eV})$. By exciting the bundles with the $785 \mathrm{~nm}$ excitation wavelength, we hypothesize that, opposite to the 
(a)

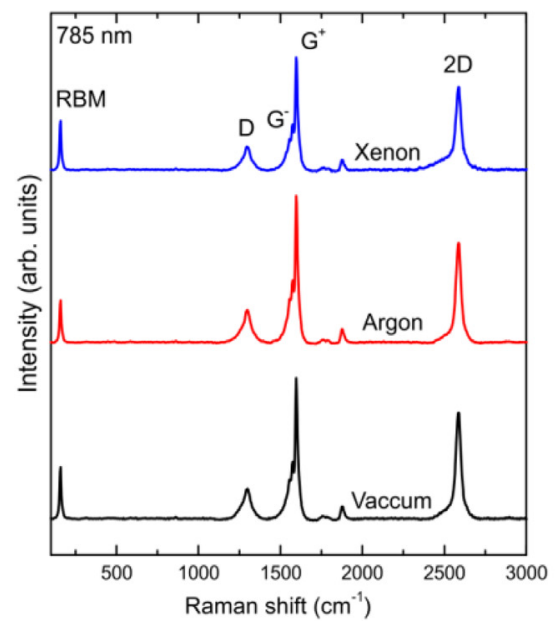

(c)

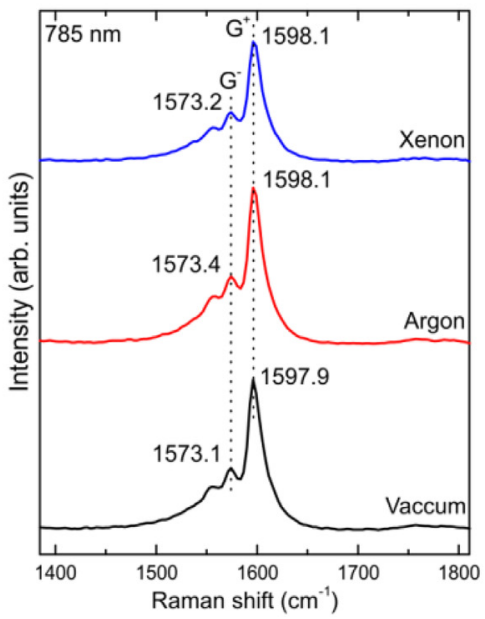

(b)

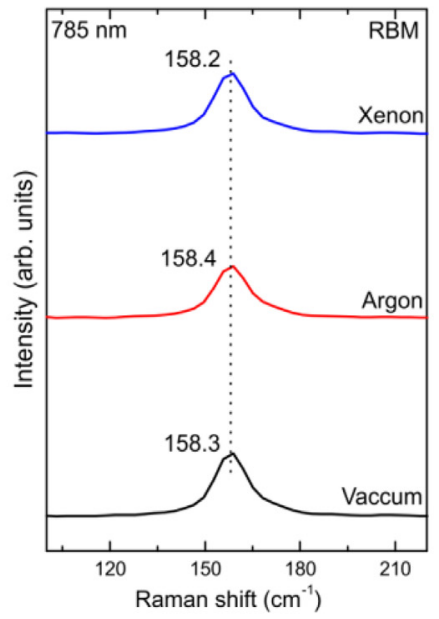

(d)

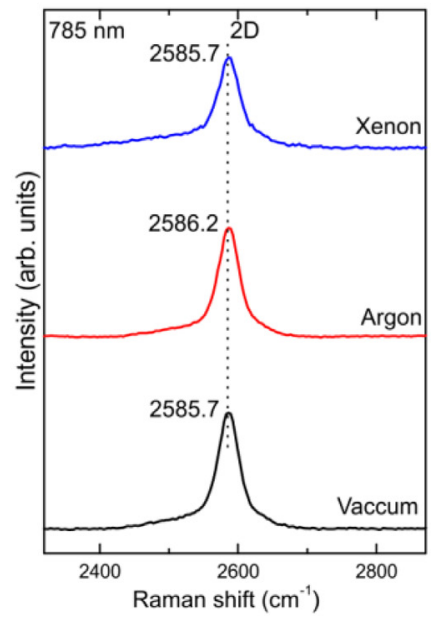

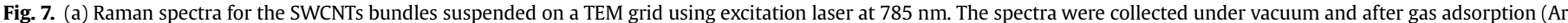

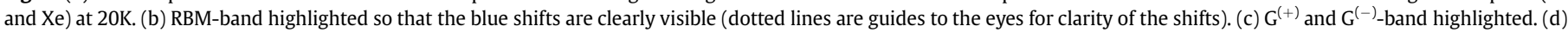
$\mathrm{G}^{\prime}$ (or 2D)-band highlighted. (A colour version of this figure can be viewed online.)

outermost tubes in the bundle, which were excited with the $488 \mathrm{~nm}$ wavelength and had a high density of adsorbates at their surface, the innermost tubes in the bundle will be less susceptible to gas adsorption. The results of this experiment are shown in Fig. 7. The absence of upshifts after gas adsorption for both $\mathrm{Ar}$ and Xe, indicates that this laser line is in resonance with nanotubes located in the internal site of the bundle in which adsorbates face more difficulties to access and get adsorbed. Thus, these inner tubes are considerably less affected by the noble gases adsorption and, therefore, less affected by the hydrostatic pressures induced by these noble gases. Indeed, these innermost tubes seem to be shielded by the outermost tubes and so we can also confirm that the outermost tubes of the bundled assembly will most likely be the species leading the reactions with external agents such as the noble gases above mentioned and other gases.

\section{Conclusions}

We have performed Raman spectroscopy measurements at 20K to study Ar and Xe adsorption on suspended SWNCT bundles. The results indicate that the adsorbents will form an external solid shell surrounding the suspended bundles. This shell contributes with a hydrostatic pressure to the system, so that the Raman frequencies are upshifted. The reported measurements are in good agreement with theoretical simulations and show a similar adsorption behavior for both noble gases. Moreover, through a convenient choice of excitation wavelengths, we were able to excite different regions in the carbon nanotube bundles to show that, while the outermost tubes are greatly affected by the adsorbents, the innermost tubes in the bundle seem to be shielded from such adsorbing atoms.

\section{Acknowledgements}

R. C. acknowledges the scholarship CNPq/PDE (234973/2014-5). P. T. A. acknowledges the College of Arts and Sciences at the University of Alabama for supporting this research through startup money funds. R.P. acknowledges FAPESP for financial support through grant 2014/15521-9. 


\section{Appendix A. Supplementary data}

Supplementary data related to this article can be found at https://doi.org/10.1016/j.carbon.2017.11.017.

\section{References}

[1] S. Iijima, Carbon nanotubes: past, present, and future, Phys. B Condens. Matter 323 (2002) 1-5.

[2] S. Iijima, T. Ichihashi, Single-shell carbon nanotubes of 1-nm diameter, Nature 363 (1993) 603-605.

[3] vol. 80, Carbon Nanotubes, Springer Berlin Heidelberg, 2001.

[4] V.N. Popov, Carbon nanotubes: properties and application, Mater. Sci. Eng. R. Rep. 43 (2004) 61-102.

[5] Q. Zhang, Carbon Nanotubes and Their Applications, CRC Press, 2012.

[6] R.E. Morris, P.S. Wheatley, Gas storage in nanoporous materials, Angew. Chem. Int. Ed. 47 (2008) 4966-4981.

[7] P.W. Barone, S. Baik, D.A. Heller, M.S. Strano, Near-infrared optical sensors based on single-walled carbon nanotubes, Nat. Mater. 4 (2005) 86-92.

[8] C.-H. Wu, et al., Trojan-horse nanotube on-command intracellular drug delivery, Nano Lett. 12 (2012) 5475-5480.

[9] D. Quiñonero, A. Frontera, P.M. Deyà, Feasibility of single-walled carbon nanotubes as materials for $\mathrm{CO}_{2}$ adsorption: a DFT study, J. Phys. Chem. C 116 (2012) 21083-21092.

[10] S. Agnihotri, J.P.B. Mota, M. Rostam-Abadi, M.J. Rood, Adsorption site analysis of impurity embedded single-walled carbon nanotube bundles, Carbon 44 (2006) 2376-2383.

[11] W. Shi, J.K. Johnson, Gas adsorption on heterogeneous single-walled carbon nanotube bundles, Phys. Rev. Lett. 91 (2003), 015504.

[12] S. Rols, et al., Argon adsorption in open-ended single-wall carbon nanotubes, Phys. Rev. B 71 (2005), 155411

[13] S. Talapatra, A.M. Migone, Existence of novel Quasi-one-dimensional phases of atoms adsorbed on the exterior surface of close-ended single wall nanotube bundles, Phys. Rev. Lett. 87 (2001), 206106.

[14] G. Stan, M.J. Bojan, S. Curtarolo, S.M. Gatica, M.W. Cole, Uptake of gases in bundles of carbon nanotubes, Phys. Rev. B 62 (2000) 2173-2180.

[15] M. Foroutan, A.T. Nasrabadi, Adsorption behavior of ternary mixtures of noble gases inside single-walled carbon nanotube bundles, Chem. Phys. Lett. 497 (2010) 213-217.

[16] L. Mandeltort, et al., Experimental and theoretical comparison of gas desorption energies on metallic and semiconducting single-walled carbon nanotubes, J. Am. Chem. Soc. 135 (2013) 7768-7776.

[17] H. Sha, R. Faller, Molecular simulation of adsorption and separation of pure noble gases and noble gas mixtures on single wall carbon nanotubes, Comput. Mater. Sci. 114 (2016) 160-166.

[18] D.-L. Chen, et al., Is there a difference in van der Waals interactions between rare gas atoms adsorbed on metallic and semiconducting single-walled carbon nanotubes? Phys. Rev. Lett. 110 (2013), 135503.

[19] Z. Wang, et al., Phase transitions of adsorbed atoms on the surface of a carbon nanotube, Science 327 (2010) 552-555.

[20] S.G. Balasubramani, D. Singh, R.S. Swathi, Noble gas encapsulation into carbon nanotubes: predictions from analytical model and DFT studies, J. Chem. Phys. 141 (2014), 184304.

[21] R. Majidi, Molecular dynamics simulation of noble gases adsorption on carbon nanotube bundles, Fuller. Nanotub. Carbon Nanostruct. 22 (2014) 520-527.

[22] H.-Y. Kim, M.W. Cole, M. Mbaye, S.M. Gatica, Phase behavior of Ar and Kr films on carbon nanotubes, J. Phys. Chem. A 115 (2011) 7249-7257.

[23] M. Arab, F. Picaud, C. Ramseyer, M.R. Babaa, F. Valsaque, E. McRae, Characterization of single wall carbon nanotubes by means of rare gas adsorption, J. Chem. Phys. 126 (2007), 054709.

[24] P. Qi, et al., Toward large arrays of multiplex functionalized carbon nanotube sensors for highly sensitive and selective molecular detection, Nano Lett. 3 (2003) 347-351.

[25] S. Chopra, K. McGuire, N. Gothard, A.M. Rao, A. Pham, Selective gas detection using a carbon nanotube sensor, Appl. Phys. Lett. 83 (2003) 2280-2282.

[26] S.M. Clark, K.-J. Jeon, J.-Y. Chen, C.-S. Yoo, Few-layer graphene under high pressure: Raman and X-ray diffraction studies, Solid State Commun. 154 (2013) 15-18.

[27] K. Filintoglou, et al., Raman spectroscopy of graphene at high pressure: effects of the substrate and the pressure transmitting media, Phys. Rev. B 88 (2013), 045418.

[28] J. Rintala, O. Herranen, A. Johansson, M. Ahlskog, M. Pettersson, Raman spectroscopy and low-temperature transport measurements of individual single-walled carbon nanotubes with varying thickness, J. Phys. Chem. C 113 (2009) 15398-15404.

[29] L. Zhang, Z. Jia, L. Huang, S. O'Brien, Z. Yu, Low-temperature Raman spectroscopy of individual single-wall carbon nanotubes and single-layer graphene, J. Phys. Chem. C 112 (2008) 13893-13900.

[30] T. Ando, Anomaly of optical phonon in monolayer graphene, J. Phys. Soc. Jpn.
75 (2006), 124701

[31] T. Ando, Anomaly of optical phonons in bilayer graphene, J. Phys. Soc. Jpn. 76 (2007), 104711.

[32] M. Lazzeri, F. Mauri, Nonadiabatic Kohn anomaly in a doped graphene monolayer, Phys. Rev. Lett. 97 (2006), 266407.

[33] L.-C. Yin, H.-M. Cheng, R. Saito, M.S. Dresselhaus, Fermi level dependent optical transition energy in metallicsingle-walled carbon nanotubes, Carbon 49 (2011) 4774.

[34] N. Caudal, A.M. Saitta, M. Lazzeri, F. Mauri, Kohn anomalies and nonadiabaticity in doped carbon nanotubes, Phys. Rev. B 75 (2007), 115423.

[35] K. Sasaki, H. Farhat, R. Saito, M.S. Dresselhaus, Kohn anomaly in Raman spectroscopy of single wall carbon nanotubes, Phys. E 42 (2010) 2005.

[36] H. Farhat, K. Sasaki, M. Kalbac, M. Hofmann, R. Saito, M.S. Dresselhaus, J. Kong, Softening of the radial breathing mode in metallic carbon nanotubes, Phys. Rev. Lett. 102 (2009), 126804.

[37] A.C.T. van Duin, S. Dasgupta, F. Lorant, W.A. Goddard, ReaxFF: a reactive force field for hydrocarbons, J. Phys. Chem. A 105 (2001) 9396-9409.

[38] K. Chenoweth, A.C.T. van Duin, W.A. Goddard, ReaxFF reactive force field for molecular dynamics simulations of hydrocarbon oxidation. J. Phys. Chem. A 112 (2008) 1040-1053.

[39] S. Plimpton, Fast parallel algorithms for short-range molecular dynamics, J. Comput. Phys. 117 (1995) 1-19.

[40] W. Humphrey, A. Dalke, K. Schulten, VMD: visual molecular dynamics, J. Mol. Graph. 14 (1996) 33-38.

[41] D. Yoon, Y.-W. Son, H. Cheong, Strain-dependent splitting of the doubleresonance Raman scattering band in graphene, Phys. Rev. Lett. 106 (2011), 155502.

[42] P.T. Araujo, et al., Nature of the constant factor in the relation between radial breathing mode frequency and tube diameter for single-wall carbon nanotubes, Phys. Rev. B 77 (2008), 241403.

[43] P.T. Araujo, A. Jorio, The role of environmental effects on the optical transition energies and radial breathing mode frequency of single wall carbon nanotubes, Phys. Status Solidi B 245 (2008) 2201-2204.

[44] P.T. Araujo, C. Fantini, M.M. Lucchese, M.S. Dresselhaus, A. Jorio, The effect of environment on the radial breathing mode of supergrowth single wall carbon nanotubes, Appl. Phys. Lett. 95 (2009), 261902.

[45] J.-X. Shi, T. Natsuki, Q.-Q. Ni, Radial buckling of multi-walled carbon nanotubes under hydrostatic pressure, Appl. Phys. A 117 (2014) 1103-1108.

[46] S. Herasati, L.C. Zhang, Elastic properties of single-walled carbon nanotube clusters: dependence on hydrostatic pressure, Comput. Mater. Sci. 86 (2014) 93-98.

[47] T.F.T. Cerqueira, S. Botti, A. San-Miguel, M.A.L. Marques, Density-functional tight-binding study of the collapse of carbon nanotubes under hydrostatic pressure, Carbon 69 (2014) 355-360.

[48] A.J. Ghandour, D.J. Dunstan, A. Sapelkin, J.E. Proctor, M.P. Halsall, High-pressure Raman response of single-walled carbon nanotubes: effect of the excitation laser energy, Phys. Rev. B 78 (2008), 125420.

[49] K. Gao, R.C. Dai, Z. Zhao, Z.M. Zhang, Z.J. Ding, Effects of pressure transmitting media on Raman features of single-walled carbon nanotubes, Solid State Commun. 147 (2008) 65-68.

[50] W. Yang, et al., Anomalous pressure behavior of tangential modes in singlewall carbon nanotubes, Phys. Rev. B 76 (2007), 033402.

[51] I.-H. Choi, P.Y. Yu, P. Tangney, S.G. Louie, Vibrational properties of single walled carbon nanotubes under pressure from Raman scattering experiments and molecular dynamics simulations, Phys. Status Solidi B 244 (2007) $121-126$.

[52] A.G. Souza Filho, A. Jorio, GeG. Samsonidze, G. Dresselhaus, M.A. Pimenta, M.S. Dresselhaus, Anna K. Swan, M.S. Ünlü, B.B. Goldberg, R. Saito, Competing spring constant versus double resonance effects on the properties of dispersive modes in isolated single-wall carbon nanotubes, Phys. Rev. B 67 (2003), 035427.

[53] M.A. Pimenta, A. Jorio, S.D.M. Brown, A.G. Souza Filho, G. Dresselhaus, J.H. Hafner, C.M. Lieber, R. Saito, M.S. Dresselhaus, Diameter dependence of the Raman D-band in isolated single-wall carbon nanotubes, Phys. Rev. B 64 (2001), 041401(R).

[54] P.T. Araujo, N.M. Barbosa Neto, H. Chacham, S.S. Carara, J.S. Soares, A.D. Souza, L.G. Cançado, A.B. Oliveira, R.J.C. Batista, E. Joselevich, M.S. Dresselhaus, A. Jorio, In situ atomic force microscopy tip-induced deformations and Raman spectroscopy characterization of single-wall carbon nanotubes, Nano Lett. 12 (2012) 4110-4116.

[55] A.P.M. Barboza, H. Chacham, B.R.A. Neves, Universal response of single-wall carbon nanotubes to radial compression, Phys. Rev. Lett. 102 (2009), 025501.

[56] D.A. McQuarrie, Statistical Mechanics, Harper and Row, New York, 1976.

[57] H. Wise, J. Oudar, Materials Concepts in Surface Reactivity and Catalysis, Dover Publications, New York, 2001.

[58] Y.W. Chung, Practical Guide to Surface Science and Spectroscopy, Academic Press, California, 2001.

[59] R.L. Amey, R.H. Cole, Dielectric constants of liquefied noble gases and methane, J. Chem. Phys. 40 (1964) 146. 